\title{
La Voce dei Pazienti Esiste un monitoraggio dei malati di ADPKD?
}

Dalla pagina Facebook di AIRP onlus, due tematiche sollevate dagli iscritti: la necessità di monitorare i pazienti per ricavare proiezioni future su terapie e trapianti e se sia opportuno o meno aspirare le cisti di maggiori dimensioni. II dibattito è sempre vivo e partecipato.

P.N.: Mi chiedevo se è possibile sapere quanti malati di ADPKD ci sono oggi in Italia, nei vari stadi della malattia (lieve o moderata IRC, pre-dialisi, dialisi, trapianto). Questi dati forse ci potrebbero far capire meglio, tra qualche anno, quanto la prevenzione e le cure attuali possano ritardare l'entrata in dialisi e/o il successo del trapianto in noi policistici.

A.M.B.: Esiste un registro dove sono iscritti i portatori del gene: di sicuro in Italia, ma credo anche a livello europeo.

P.O.: Intanto ti posso dire che ogni seduta di dialisi costa allo Stato 400 euro. Considera che sono tre sedute a settimana... e il conto è fatto. Chiaramente le grandi multinazionali farmaceutiche ci tengono a fornire macchine, linee, filtri, liquidi e così via per le dialisi. Si parla di milioni di euro! Questo, secondo me, è il motivo per cui non esiste ancora una cura. Olé.

P.N.: Non volevo entrare negli aspetti economici (anche se determinanti e su cui si potrebbero aprire un sacco di discussioni), ma volevo soltanto domandare se qualcuno monitorizza la situazione delle cure offerte specificatamente ai malati di ADPKD qui in Italia, per capire se, nel corso degli anni, vi possono essere stati dei miglioramenti in termini numerici per verificare l'efficacia delle cure preventive e dei trapianti, evitando il ricorso alla dialisi. Naturalmente, il punto di partenza è quello di un registro dei portatori del gene ma occorre precisare che non tutti, fortunatamente, vanno in insufficienza renale, se non iñ età avanzata, e, addirittura, non hanno bisogno di ricorrere a cure.

L.S.P.: Non esistono, in Italia, registri specifici su ADPKD, ma si sta studiando il problema. Studi scientifici dimostrano che l'entrata in dialisi di questi pazienti è posticipata in media di dieci anni.

P.N.: Ecco, la Presidente ha dato due risposte importanti: che non esiste attualmente un registro, ma che gli specialisti medici tengono sotto controllo i dati e che, con le cure opportune (stili di vita, diete, cure con nuovi farmaci), si può ritardare di molto il ricorso alla dialisi. Grazie, speriamo che un attento monitoraggio sappia dare indicazioni più ottimistiche per il futuro.

L.S.P.: Ci stiamo lavorando moltissimo.

Togliere le cisti? Forse meglio di no.

F.L.P.: Buongiorno. Sapete se le cisti, soprattutto quelle di grandi dimensioni, possono essere aspirate? Parlando con un medico qualche giorno fa, lui sottolineava addirittura l'importanza di farlo il prima possibile, per far lavorare meglio i reni, prima che vengano compromessi. cosa ne pensate?

A.M.: Che non hai parlato con un medico...
M.R.P.: lo l'ho fatto! Al Cannizzaro di Catania e, per un paio d'anni, sono stata bene. II problema è che poi si riformano le stese cisti che hanno aspirato. Ora lo sconsigliano, perché inutile è comporta dei rischi. Al Cannizzaro è una procedura giornaliera...

P.M.: lo, veramente, avrei voluto anche eliminarle, ma mi è stato detto che ne avrei avuto solo danni. Perciò non l'ho mai fatto. Ma le mie cisti sono progredite in tutti e due i reni e oggi sono in dialisi peritoneale.

M.B.: A me hanno sempre detto di non toccarle. A volte quelle più grosse si sono rotte da sole.

P.M.: Si, è súccesso anche a me, due volte nell'arco di 30 anni. Normalmente avevo fitte giornaliere dolorosissime, che riuscivo ad alleviare con la tachipirina.

L.P.: Da ignorante in materia, io so che le cisti non si devono togliere: primo, perché si riformano e, secondo, perché si fa subire al proprio corpo e ai reni uno stress pesantissimo con un intervento che, in sostanza, è inutile.

R.M.: È una procedura superata, inutile oltre che dannosa: le cisti recidivano e vi è un alto rischio di complicanze emorragiche e infettive.

B.F.: Se fosse una terapia sensata, avremmo già risolto il problema!!!

F.L.P.: Scusate se insisto, qualche parere medico è possibile averlo? Credo possa essere un argomento interessante.

A.D.C.: Hai ragione, ma le risposte che leggi sono quello che vari medici ci dicono e suggeriscono di fare...

D.M.: A me hanno detto che le cisti non si devono toccare. Ho sentito diversi pareri medici e tutti hanno detto la stessa cosa!

D.V.: Anche a me hanno detto che non vanno toccate, perché si riformano e perché non è bene andarle a stuzzicare: potrebbero verificarsi danni peggiori.

M.G.: A Bologna sconsigliano di toccare le cisti, salvo in casi disperati, quando compromettono la funzionalità di altri organi o non si riesce più a mangiare. Piuttosto, si consiglia di asportare i reni completamente. Per il fegato, è un altro discorso: non si può togliere, ma si può fare una resezione, cioè tagliarne un pezzo. II Prof. Pinna, che fa i trapianti multipli, ha spiegato in un convegno che solo certe cisti, non quelle emorragiche, si possono toccare senza problemi. Se, invece, sono emorragiche, si rischia di morire per infezione o dissanguamento.

B.M.: Io sono in cura al Cannizzaro di Catania e la Dr.ssa Marcantoni evita proprio l'aspirazione delle cisti, non esiste affatto. Può accadere quando sono di dimensioni tali da far sentire molto dolore, ma è un caso estremo. 